\title{
Feline Hypertrophic Cardiomyopathy: A Spontaneous Large Animal Model of Human HCM
}

\author{
Lisa M. Freeman ${ }^{\mathrm{a}, ~ d}$, John E. Rush ${ }^{\mathrm{a}}$, Joshua A. Stern ${ }^{\mathrm{b}}$, Gordon S. Huggins ${ }^{\mathrm{c}}$, \\ Martin S. Maron ${ }^{\mathrm{c}}$
}

\begin{abstract}
Hypertrophic cardiomyopathy (HCM) is a common disease in pet cats, affecting $10-15 \%$ of the pet cat population. The similarity to human HCM, the rapid progression of disease, and the defined and readily determined endpoints of feline HCM make it an excellent natural model that is genotypically and phenotypically similar to human HCM. The Maine Coon and Ragdoll cats are particularly valuable models of HCM because of myosin binding protein- $\mathrm{C}$ mutations and even higher disease incidence compared to the overall feline population. The cat overcomes many of the limitations of rodent HCM models, and can provide enhanced translation of information from in vitro and induced small animal models to human clinical trials. Physicians and veterinarians working together in a collaborative and interdisciplinary approach can accelerate the discovery of more effective treatments for this and other cardiovascular diseases affecting human and veterinary patients.
\end{abstract}

Keywords: Hypertrophic cardiomyopathy; Cats; Congestive heart failure; Natural animal model; Arterial thromboembolism

\section{Introduction}

Companion animals and humans frequently suffer from similar diseases, such as heart disease, cancer, kidney disease, arthritis, diabetes, and obesity. Prevalence rates can be remarkably similar across species. Obesity, for example, affects similar numbers of humans, dogs, and cats. However, because of selective

Manuscript submitted July 18, 2017, accepted July 28, 2017

aDepartment of Clinical Sciences, Cummings School of Veterinary Medicine at Tufts University, 200 Westboro Road, North Grafton, MA, USA

${ }^{b}$ Department of Medicine and Epidemiology, School of Veterinary Medicine, University of California Davis, CCAH Room 258, One Shields Avenue, Davis, CA, USA

'Department of Medicine, Tufts Medical Center, 800 Washington Street, Boston, MA 02111, USA

${ }^{\mathrm{d}}$ Corresponding Author: Lisa Freeman, Department of Clinical Sciences, Cummings School of Veterinary Medicine at Tufts University, 200 Westboro Road, North Grafton, MA 01536, USA. Email: lisa.freeman@tufts.edu

doi: https://doi.org/10.14740/cr578w breeding to achieve certain physical or behavioral traits, pet dogs and cats can have even higher risk for some diseases. For example, $>50 \%$ of Doberman Pinscher dogs develop dilated cardiomyopathy (DCM) [1] and nearly $100 \%$ of Cavalier King Charles Spaniels develop degenerative mitral valve disease (DMVD) [2]. These naturally occurring diseases in pets are much more representative of the human disease than induced rodent models, so studying the disease in companion animals provides an innovative and effective approach for accelerating translational research that can ultimately benefit both humans and animals. Diagnosis and treatment of companion animal diseases can be nearly identical to that of humans, with similar diagnostic tests (e.g., advanced imaging, laboratory testing, and genetic testing) and treatments (e.g., medications, interventional cardiology techniques, and specialized surgical techniques). These studies of naturally occurring diseases can be conducted in genetically defined colonies or through clinical trials in pet dogs and cats. Both approaches can provide an important stepping stone between basic science research and human clinical trials that can reduce the all-too-common translational failures.

\section{Heart Disease Affects More Than Humans}

Heart disease is one of the most common diseases of pet dogs and cats, affecting $10-15 \%$ of all dogs and cats $[3,4]$. The most common diseases in dogs are DMVD and cardiomyopathies (primarily DCM or arrhythmogenic right ventricular cardiomyopathy), but they also can be affected by a variety of congenital heart defects and pericardial diseases. Prevalence rates are even higher in certain breeds, with distinct breed predispositions for heart diseases in both dogs and cats. For example, large and giant breed dogs, such as Doberman Pinschers, Great Danes, and Irish Wolfhounds, are at higher risk for DCM, while small- to medium-size breeds, such as Cavalier King Charles Spaniels, Dachshunds, and Poodles are predisposed to DMVD. Cats are most commonly affected by hypertrophic cardiomyopathy $(\mathrm{HCM})$, with a prevalence of $10-15 \%$ in the general pet cat population [4]. However, breeds such as the Maine Coon cat, Persian, Ragdoll, and Sphynx are at higher risk. Similar to humans, sarcomeric protein mutations are implicated in the pathogenesis of feline HCM.

Although heart disease is common in dogs and cats, atherosclerosis is notably absent. This is related, at least in part, to 


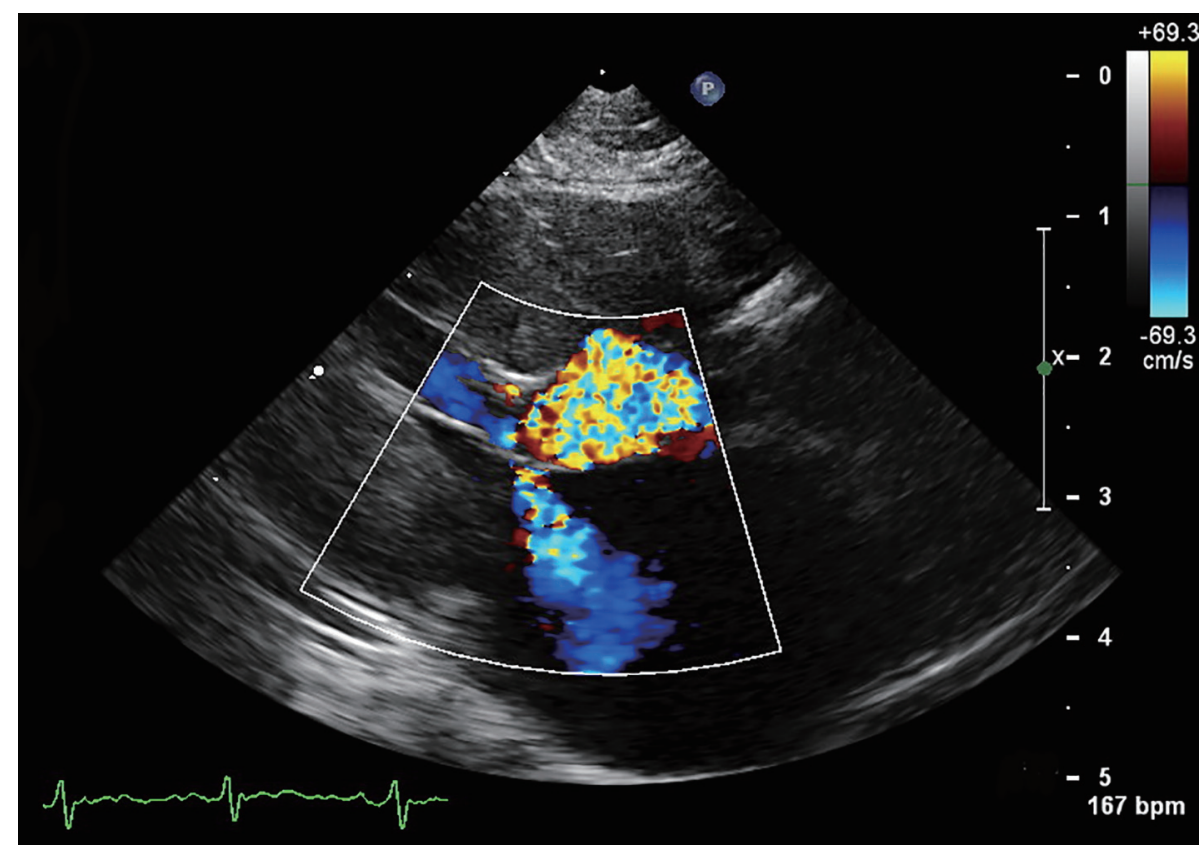

Figure 1. A right parasternal echocardiogram with color flow Doppler from a 7-year-old cat with hypertrophic obstructive cardiomyopathy. Mitral regurgitation and left ventricular outflow tract obstruction secondary to systolic anterior motion of the mitral valve are seen. This patient has significant left ventricular hypertrophy of the interventricular septum and free wall.

the fact that these species have high high-density lipoprotein concentrations.

\section{HCM: Challenges and Solutions}

In humans, there are now known to be $>1,500$ causative mutations associated with HCM, with the vast majority being identified in one of 11 sarcomeric genes. Nonetheless, up to $30 \%$ of human patients with HCM have no identified mutation and there is wide phenotypic variation, even among patients with the same genetic mutation. Rodent models of HCM (e.g., transgenic or naturally occurring mutations) are useful in the study of basic mechanisms, but have many limitations for translational breakthroughs. While rodent models may have the same genetic mutation as in human HCM, the phenotype is typically not the same as in human HCM. For example, most mouse models expressing myosin binding protein-C (MYBPC) mutations do not develop myocardial hypertrophy, the defining trait in humans (and cats) with HCM, or share features of symptoms, clinical course, or response to treatment. These partial models therefore may have limited predictive value in translating information to humans due to species differences (e.g., anatomy, metabolic rate, lifespan, and response to treatment). These limitations can result in translational failures when drugs showing promise in rodent models are tested in human clinical trials. This underscores the importance of having relevant preclinical models that can fill the gap between induced rodent models and human clinical medicine.

HCM occurs spontaneously and frequently in pet cats, and the feline disease is remarkably similar to the human disease $[5,6]$. There is a male predisposition for HCM in both species and clinical signs are similar, although more common and more severe in cats than in humans. The most common presentations in cats are sudden death or syncope, congestive heart failure $(\mathrm{CHF})$, and arterial thromboembolism (ATE). Many cats with $\mathrm{HCM}$ are identified incidentally when a cardiac murmur is auscultated. Once diagnosed with HCM, most cats eventually die from $\mathrm{CHF}$, sudden death, or ATE, while a smaller proportion remains subclinical. The same diagnostic tests used in humans (e.g., N-terminal B-type natriuretic peptide, radiography, electrocardiography, and echocardiography) are used to diagnose cats with HCM (Fig. 1), and similar medications are used to manage the disease. Finally, feline HCM is very similar to human HCM grossly (Fig. 2) and on histopathology [6]. One difference is that feline HCM is a more severe and quickly progressive disease than in humans, an advantage when studying outcomes. Clinical studies have shown a median survival time in cats with HCM ranging from 92 to 2,153 days, depending on the predominant clinical signs of the population studied (i.e., asymptomatic vs. CHF vs. ATE) [7-9]. Reported median survival times for cats with $\mathrm{HCM}$ and $\mathrm{CHF}$, for example, range from only 92 to 563 days [7-9].

\section{Treatment of Feline HCM}

Treatment of feline HCM can be divided into the preclinical and clinical (CHF or ATE) time periods. As in humans, despite advances in the understanding of HCM, no preclinical therapy has demonstrated the ability to slow disease progression or reduce ventricular hypertrophy in cats. The most commonly employed therapies include cardioprotective strategies such as beta-blockade (especially when left ventricular outflow 


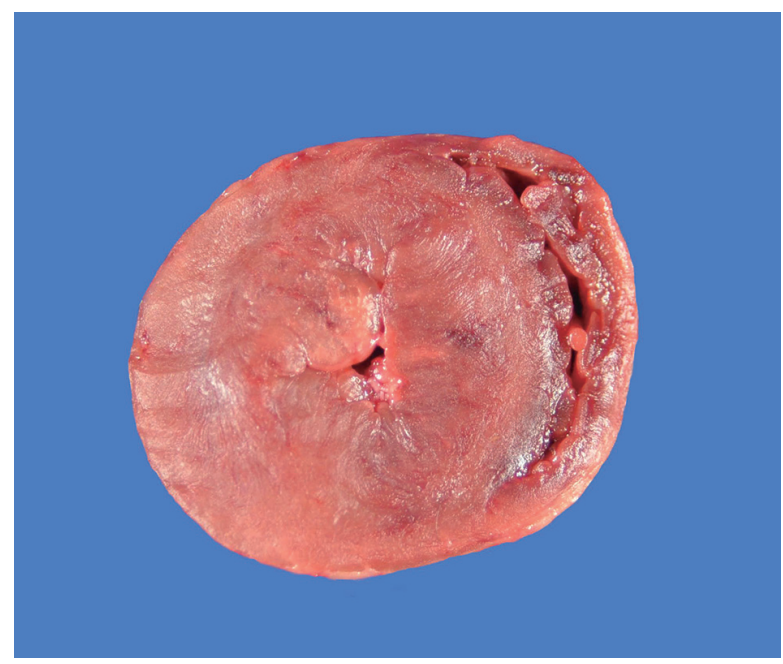

Figure 2. A gross pathology specimen from a 5-year-old cat with hypertrophic cardiomyopathy. The heart is shown in a transverse plane at the level of the papillary muscles in the left ventricle. Severe concentric left ventricular hypertrophy is evident with dramatic reduction in the size of the left ventricular lumen.

tract obstruction is observed), ACE-inhibition, and antiplatelet drugs. Some promise with novel, targeted therapies is on the horizon as sarcomeric modulating drugs are being investigated. The feline HCM model has already served as a bridge between research in rodents and human clinical trials with one such novel drug trial in cats with HCM [10]. Beta receptor and ACE gene polymorphisms are described in cats and their role in the response to therapy and progression of HCM, and provide another opportunity for interdisciplinary research.

In cats with HCM and CHF, the above-mentioned treatments are expanded to include diuretics, such as furosemide, and sometimes pimobendan, a drug with positive inotropic and vasodilatory effects. ATE is common in cats with HCM, but therapy and prognosis for feline patients with ATE are limited and represent another area of future research.

\section{Genetics of Feline HCM}

Two separate MYBPC mutations, which represent the second most common human gene affected, have been identified in two cat breeds, the Maine Coon cat and the Ragdoll [11]. In Maine Coon cats, for example, an MYBPC mutation (A31P) has been detected in approximately $22-42 \%$ of Maine Coon cats, while a separate MYBPC mutation (R820W) has been identified in $27 \%$ of Ragdoll cats [11]. The R820W mutation was later identified in a human family with HCM and thus represents a shared genetic etiology between cats and humans [12]. But Maine Coon cats without the A31P mutation also can develop HCM, underscoring the presence of unidentified mutations. In these cats and in the majority of cats with HCM, no genetic etiology has been identified.

Mutations in humans may be pathogenic or non-pathogenic. And similar to humans, the mutations identified in cats appear to have incomplete penetrance. In Maine Coon cats with a
MYBPC mutation, penetrance is reported to be $6-8 \%$ in $\mathrm{A} 31 \mathrm{P}$ heterozygotes and $58-80 \%$ in homozygotes [11]. However, in both humans and cats, some patients with mutations have minimal cardiac changes, while some with the same mutation and even in the same family have severe hypertrophy with advanced clinical signs or sudden death. This variable genotypephenotype relationship suggests that there are other genetic or environmental modifiers of phenotype.

Nutrient-gene interactions (nutrigenomics) may provide a key to understanding of the phenotypic variability of HCM and an opportunity for improved therapeutic targets and nutritional strategies to reduce morbidity and mortality. Pet cats with HCM are larger in size, more likely to be overweight, have higher concentrations of glucose and IGF-1, and grow more quickly in early life compared to healthy controls [13]. This suggests that early growth and nutrition may influence the development and course of HCM in genetically predisposed cats. Studying the role of nutrigenomics in humans with HCM is warranted.

\section{Opportunities for Collaboration}

Instead of approaching HCM from the traditional silos of individual disciplines, a collaborative and interdisciplinary approach can accelerate translation of research into effective medical innovations for humans with this disease. Studying $\mathrm{HCM}$ in cats with naturally occurring disease can enhance translational efficiency and effectiveness between basic science research and human clinical trials. At the same time, discoveries in humans can spur advances in the treatment of cats with this disease.

Cats with HCM could serve as a good model for human HCM to study novel drugs, disease progression, or development of CHF, but would be less than ideal for studying sudden death, since this is not a common occurrence in cats with HCM. Veterinary cardiologists can help to determine the optimal study design based on the research question and outcomes of interest. Some specific opportunities to collaborate or learn more are listed below. 1) Feline HCM can be studied as a model for human HCM either as genetically defined cats in a colony or through clinical trials in pet cats (which typically are not screened for mutations unless they are of certain specific breeds, such as the Maine Coon cat or Ragdoll). 2) Find out about current clinical trials in companion animals. Animal clinical trials can be shorter and less expensive since dogs and cats with heart disease often reach endpoints (e.g., heart failure) earlier than humans. There are numerous, ongoing clinical trials in a variety of naturally occurring diseases in companion animals, including HCM. Clinical trials in companion animals must undergo approval (by an Institutional Animal Care and Use Committee or similar body) and pet owners must sign an informed consent form before volunteering to enroll their pets, along with a variety of other safeguards for both the pet owner and the pet. A list of some of the current clinical trials across the United States can be found on the American Veterinary Medical Association's Clinical Trials Registry (https:// ebusiness.avma.org/aahsd/study_search.aspx) and on indi- 
vidual veterinary school website's (e.g., http://vet.tufts.edu/ cvmc/clinical-studies/, http:/www.vetmed.ucdavis.edu/clinicaltrials/current_trials/index.cfm). 3) Read about published research using natural animal models of HCM and other cardiovascular diseases. Many veterinary studies are indexed in Medline and can be accessed via Pubmed or Ovid. However, some veterinary studies may only be indexed in $\mathrm{CAB} A \mathrm{~A}$ stracts. Therefore, one must deliberately seek out studies using natural animal models. 4) Invite veterinary cardiologists to give sessions at cardiology meetings. The interactions can be enlightening, both from a clinical and research perspective. 5) Collaborate with veterinarians and veterinary cardiologists in research. An interdisciplinary approach provides important advantages for optimal study design. Despite the many similarities between human and feline HCM, there are some important metabolic, nutritional, and anatomic differences across species that are important to understand. 6) Reach out to a veterinary cardiologist to learn more. There are currently $>300$ board-certified veterinary cardiologists in the United States (and $>500$ worldwide). Cardiologists are located at all 30 of the United States veterinary schools and in many private specialty practices across the country. Listings of board-certified veterinary cardiologists can be found online (http://find. vetspecialists.com).

\section{Grant Support}

Supported by a grant from the Clinical and Translational Science Award One Health Alliance and by the National Center for Advancing Translational Sciences, National Institutes of Health, Award Number UL1TR001064. The content is solely the responsibility of the authors and does not necessarily represent the official views of the NIH.

\section{References}

1. Wess G, Schulze A, Butz V, Simak J, Killich M, Keller LJ, Maeurer J, et al. Prevalence of dilated cardiomyopathy in Doberman Pinschers in various age groups. J Vet Intern Med. 2010;24(3):533-538.

2. Pedersen HD, Lorentzen KA, Kristensen BO. Echocardiographic mitral valve prolapse in cavalier King Charles spaniels: epidemiology and prognostic significance for regurgitation. Vet Rec. 1999;144(12):315-320.
3. Buchanan JW. Prevalence of cardiovascular disorders. In: Fox PR, Sisson DD, Moise NS (eds). Textbook of canine and feline cardiology, 2nd ed. Philadelphia: WB Saunders, 1999:457-470.

4. Payne JR, Brodbelt DC, Luis Fuentes V. Cardiomyopathy prevalence in 780 apparently healthy cats in rehoming centres (the CatScan study). J Vet Cardiol. 2015;17(Suppl 1):S244-257.

5. Kittleson MD, Meurs KM, Munro MJ, Kittleson JA, Liu SK, Pion PD, Towbin JA. Familial hypertrophic cardiomyopathy in maine coon cats: an animal model of human disease. Circulation. 1999;99(24):3172-3180.

6. Fox PR. Hypertrophic cardiomyopathy. Clinical and pathologic correlates. J Vet Cardiol. 2003;5(2):39-45.

7. Atkins CE, Gallo AM, Kurzman ID, Cowen P. Risk factors, clinical signs, and survival in cats with a clinical diagnosis of idiopathic hypertrophic cardiomyopathy: 74 cases (1985-1989). J Am Vet Med Assoc. 1992;201(4):613-618.

8. Rush JE, Freeman LM, Fenollosa NK, Brown DJ. Population and survival characteristics of cats with hypertrophic cardiomyopathy: 260 cases (1990-1999). J Am Vet Med Assoc. 2002;220(2):202-207.

9. Payne JR, Borgeat K, Connolly DJ, Boswood A, Dennis S, Wagner T, Menaut $\mathrm{P}$, et al. Prognostic indicators in cats with hypertrophic cardiomyopathy. J Vet Intern Med. 2013;27(6):1427-1436.

10. Stern JA, Markova S, Ueda Y, Kim JB, Pascoe PJ, Evanchik MJ, Green EM, et al. A small molecule inhibitor of sarcomere contractility acutely relieves left ventricular outflow tract obstruction in feline hypertrophic cardiomyopathy. PLoS One. 2016;11(12):e0168407.

11. Kittleson MD, Meurs KM, Harris SP. The genetic basis of hypertrophic cardiomyopathy in cats and humans. J Vet Cardiol. 2015;17(Suppl 1):S53-73.

12. Ripoll Vera T, Monserrat Iglesias L, Hermida Prieto M, Ortiz M, Rodriguez Garcia I, Govea Callizo N, Gomez Navarro C, et al. The R820W mutation in the MYBPC3 gene, associated with hypertrophic cardiomyopathy in cats, causes hypertrophic cardiomyopathy and left ventricular non-compaction in humans. Int $\mathrm{J}$ Cardiol. 2010;145(2):405-407.

13. Freeman LM, Rush JE, Meurs KM, Bulmer BJ, Cunningham SM, et al. Body size and metabolic differences in Maine Coon cats with and without hypertrophic cardiomyopathy. J Fel Med Surg. 2013;15:74-80. 